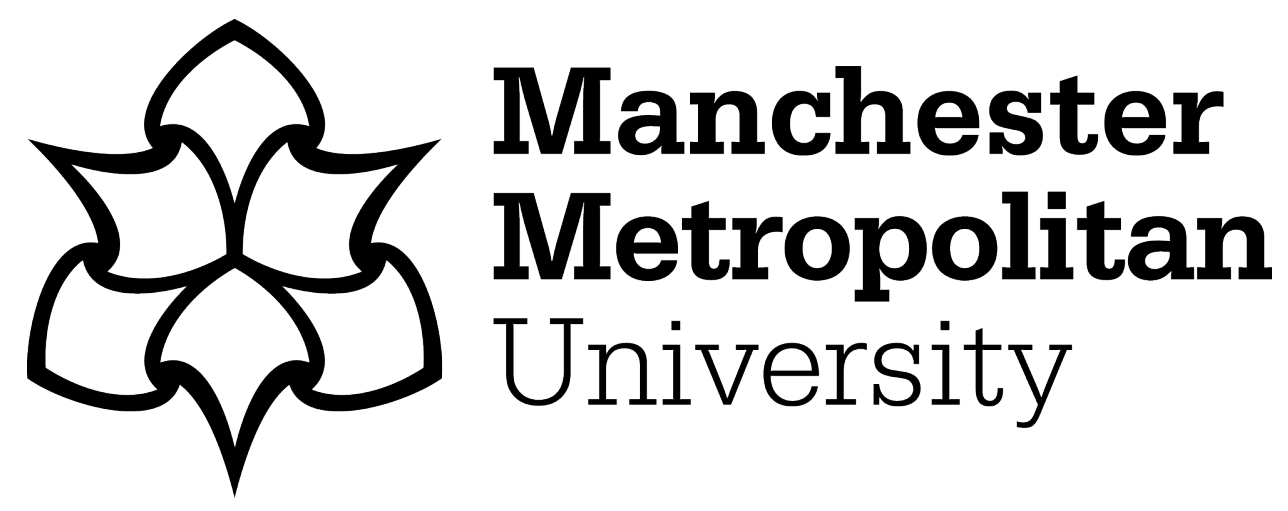

Gauthier, Joseph A, King, Laurie A ORCID logoORCID: https://orcid.org/0000-0002-0772-2378, Stults, Faith Tucker, Flores, Raul A, Kibsgaard, Jakob, Regmi, Yagya N, Chan, Karen and Jaramillo, Thomas F (2019) Transition Metal Arsenide Catalysts for the Hydrogen Evolution Reaction. The Journal of Physical Chemistry C, 123 (39). pp. 24007-24012. ISSN 1932-7447

Downloaded from: https://e-space.mmu.ac.uk/624260/

Version: Accepted Version

Publisher: American Chemical Society (ACS)

DOI: https://doi.org/10.1021/acs.jpcc.9b05738

Please cite the published version 


\title{
Transition Metal Arsenide Catalysts for the Hydrogen Evolution Reaction
}

Joseph A. Gauthier*, 1,2 Laurie A. King*, ${ }^{1,2}$ Faith Tucker Stults, ${ }^{1,2}$ Raul A. Flores, ${ }^{1,2}$ Jakob Kibsgaard, ${ }^{3}$ Yagya N. Regmi, ${ }^{4}$ Karen Chan, ${ }^{2}$ Thomas F. Jaramillo ${ }^{\dagger 1,2}$

${ }^{1}$ SUNCAT Center for Interface Science and Catalysis, SLAC National Accelerator Laboratory, 2575 Sand Hill Road, Menlo Park, California 94025, United States

${ }^{2}$ SUNCAT Center for Interface Science and Catalysis, Department of Chemical Engineering, Stanford University, Stanford, California 94305, United States

${ }^{3}$ Department of Physics, Technical University of Denmark, Fysikvej, Building 312, Dk-2800 Kongens Lyngby, Denmark

${ }^{4}$ Lawrence Berkeley National Laboratories, Energy Technologies Area, 1 Cyclotron Road, MS 0700108, Berkeley, California 94720, United States

* indicates these authors contributed equally to this work

\begin{abstract}
We report, to our knowledge for the first time, a combined experimental and density functional theory (DFT) investigation into the activity and stability of cobalt, molybdenum, and copper arsenides as catalysts for the hydrogen evolution reaction (HER). We find CoAs and MoAs to be the most active arsenide materials. We discuss the trends between calculated surface vacancy formation energies and catalyst stability. Using a simple thermodynamic model of HER activity, we find consistent trends between hydrogen binding free energy and the experimentally observed activity.
\end{abstract}

\section{Introduction}

Hydrogen is an essential chemical feedstock produced globally at over 60 million tons per year for use in a wide variety of processes, including oil refining and thermochemical ammonia synthesis via the Haber-Bosch process. ${ }^{1}$ Furthermore, hydrogen offers a sustainable pathway for the storage of renewable energy via technologies such as polymer electrolyte membrane fuel cells (PEMFC) ${ }^{2-4}$. However, $96 \%$ of hydrogen is currently produced from fossil fuels ${ }^{5}$ through processes such as the steam reforming of methane, which introduces large amounts of carbon dioxide into the atmosphere. One promising route for the sustainable production of hydrogen is through the coupling of renewable sources (e.g. wind and solar) with electrochemical water splitting.

Electrolytic hydrogen production proceeds via the hydrogen evolution reaction (HER) coupled with the oxygen evolution reaction (OER). Whereas alkaline-based electrolyzer technologies can utilize highly active earth-abundant catalysts, proton exchange membrane (PEM) electrolysers require precious metal-based catalysts due to the highly corrosive acidic conditions. ${ }^{3}$ The most active electrocatalysts for the HER are platinum based materials, which 
require overpotentials of $<50 \mathrm{mV}$ to reach $10 \mathrm{~mA} \mathrm{~cm}^{-2}{ }^{6-8}$ However, due to the high cost and scarcity of platinum, ${ }^{9}$ alternative non-precious metal based catalysts are being investigated, such as transition metal dichalcogenides, ${ }^{10-15}$ carbides, ${ }^{16-18}$ and borides. ${ }^{17}$ More recently, transition metal phosphides have received significant attention as excellent non-precious metal HER catalysts. ${ }^{19-22}$ Anion mixing, such as forming molybdenum phosphosulfide, has been proven as another route towards designing more active catalysts. ${ }^{21}$ Just as transition metal selenides have been found to be active for the HER following the success of $\mathrm{MoS}_{2},{ }^{15,23}$ with selenium one period beneath sulfur, one period beneath phosphorous lies arsenic. Transition metal arsenides (TMAs) are an unexplored family of materials for HER catalysts.

In this paper, we investigate the performance of transition metal arsenides for the HER using a combined experimental and computational approach. In this exploratory work, we synthesized and characterized three thin film transition metal arsenides, specifically, CoAs, MoAs, and $\mathrm{Cu}_{3}$ As. The films are shown to be crystalline and nanostructured. Using density functional theory (DFT), we identify the thermodynamically stable surface facets and surface terminations of these transition metal arsenides. We find trends in the experimental activities to be consistent with a simple thermodynamic picture of HER activity, where the binding free energy of hydrogen, $\Delta G_{\mathrm{H}}$ is a descriptor of HER activity, as has been found previously with metals, ${ }^{24-26}$ metal alloys, ${ }^{7}$ as well as a wide variety of ionic compounds. ${ }^{10,11,16,19}$

\section{Experimental}

The general synthesis of transition metal arsenides was analogous to work previously published for the synthesis of transition metal phosphides. ${ }^{19,21}$ In brief, cobalt nitrate hexahydrate $\left(\mathrm{Co}\left(\mathrm{NO}_{3}\right)_{2} \cdot 6 \mathrm{H}_{2} \mathrm{O}\right)$, Sigma-Aldrich), ammonium heptamolybdate $\left(\left(\mathrm{NH}_{4}\right)_{6} \mathrm{Mo}_{7} \mathrm{O}_{24} \cdot 4 \mathrm{H}_{2} \mathrm{O}\right)$ and sodium arsenate dibasic heptahydrate $\left(\mathrm{Na}_{2} \mathrm{HAsO}_{4} .7 \mathrm{H}_{2} \mathrm{O}\right.$, Sigma) were dissolved in Millipore water to make stoichiometric $0.0625 \mathrm{M}$ of transition metal arsenides in the general formula of MoAs, $\mathrm{Cu}_{3} \mathrm{As}$, and CoAs. Where necessary, a few drops of nitric acid $(6 \mathrm{M})$ were added to the solution to ensure complete dissolution of the metal salts. To prepare the films, the solutions were drop cast onto titanium foil $(0.5 \mathrm{~cm} \times 0.5 \mathrm{~cm})$ on a hotplate at approximately $50{ }^{\circ} \mathrm{C}$ to prepare samples with a final loading of $0.25 \mathrm{mg} . \mathrm{cm}^{-2}$ transition metal arsenide. The foils were subsequently annealed at $750{ }^{\circ} \mathrm{C}$ in a forming gas atmosphere.

Materials characterization. X-ray diffraction (XRD) was performed using a Bruker D8 Venture with a $\mathrm{Cu}(\mathrm{k} \alpha)$ source. Electrochemical testing was performed in a standard 3-electrode configuration. A graphite carbon rod was used as a counter electrode and a mercury/mercury sulfate reference (Hache) electrode was used with 0.5 M sulfuric acid (Aldrich, 99.999\%) electrolyte with an $\mathrm{H}_{2}$ purge for all testing. The reference electrode was calibrated to the reversible hydrogen electrode (RHE). All CVs were iR-corrected using electrochemical impedance spectroscopy to calculate the uncompensated resistance.

Computational Methods. Density functional theory (DFT) in conjunction with the computational hydrogen electrode ${ }^{27}$ (CHE) was used to determine hydrogen adsorption and surface energies. All calculations were performed using the Bayesian Error Estimation Functional $^{28}$ (BEEF) in Quantum Espresso ${ }^{29}$ interfaced through the Atomic Simulation Environment ${ }^{30}$ (ASE). The core electrons of copper, molybdenum, cobalt, arsenic, oxygen, and hydrogen are represented in this work by ultrasoft pseudopotentials, ${ }^{31-33}$ while the valence electrons have been expanded as plane waves with a kinetic energy cutoff of $600 \mathrm{eV}$ and a charge density cutoff of $6000 \mathrm{eV}$. Further details of the simulation cells can be found in the supporting information. 
Predominant surface facets and terminations of $\mathrm{Cu}_{3} \mathrm{As}$, MoAs, and CoAs were determined using the Bravais-Friedel-Donnay-Harker (BFDH) algorithm ${ }^{34}$ along with surface energy calculations. For MoAs and CoAs, the most stable facets were (001), (010), and (100), while for $\mathrm{Cu}_{3} \mathrm{As}$, the most stable facets were (001) and (110). The surface energies for these facets were all within a range of $0.1 \mathrm{eV}$, so we considered the activity on all of them. The surface energies are shown in Figure S1. Vacancy formation energies for each surface and facet were determined, using bulk metal as the reference for metal atoms, and $\mathrm{AsH}_{3}(\mathrm{~g})^{35}$ as the reference for As atoms, shown in Equation 2:

$$
E_{\mathrm{vac}}=E_{\text {surf,vac }}-\left(E_{\text {surf }}-E_{\text {ref }}\right)
$$

Where $E_{\mathrm{vac}}$ refers to the vacancy formation energy, $E_{\text {surf,vac }}$ refers to the energy of the surface with a vacancy, $E_{\text {surf }}$ refers to the energy of the surface without a vacancy, and $E_{\text {ref }}$ refers to the reference energy of the atom removed to create the vacancy.

Formation energies of various arsenide compounds were calculated and compared with tabulated literature values, ${ }^{36}$ and were found to be in reasonable agreement (Figure S2 in supporting information), suggesting the use of GGA-DFT to be appropriate for these systems. Following Kibsgaard et al. ${ }^{19}$, we assume that the HER activity arises predominantly from sites where hydrogen adsorption energies, $\Delta G_{\mathrm{H}}$, are closest to zero.

\section{Results and Discussions}

Drop casting was used to prepare thin films of $\mathrm{CoAs}$, MoAs, and $\mathrm{Cu}_{3} \mathrm{As}$ on Ti foil substrates. The transition metal arsenide (TMAs) catalysts synthesized in a forming gas environment at $750{ }^{\circ} \mathrm{C}$. X-ray diffraction (XRD) patterns in Figure 1 and Table S1 show that crystalline metal arsenides were obtained. The predominant phases synthesized were CoAs, MoAs, and $\mathrm{Cu}_{3} \mathrm{As}$. $\mathrm{CoAs}$ and MoAs adopt orthorhombic crystal structure while $\mathrm{Cu}_{3} \mathrm{As}$ is hexagonal. In each diffractogram the Ti substrate peaks are clearly visible (see supporting information for further details). The morphology of the synthesized TM-As films was probed by scanning electron microscopy (SEM) (Figure 2a-c). The thin films appear relatively dense, with a nanostructured surface. Feature sizes are generally consistent across the three films, with no distinct difference in morphology observed.
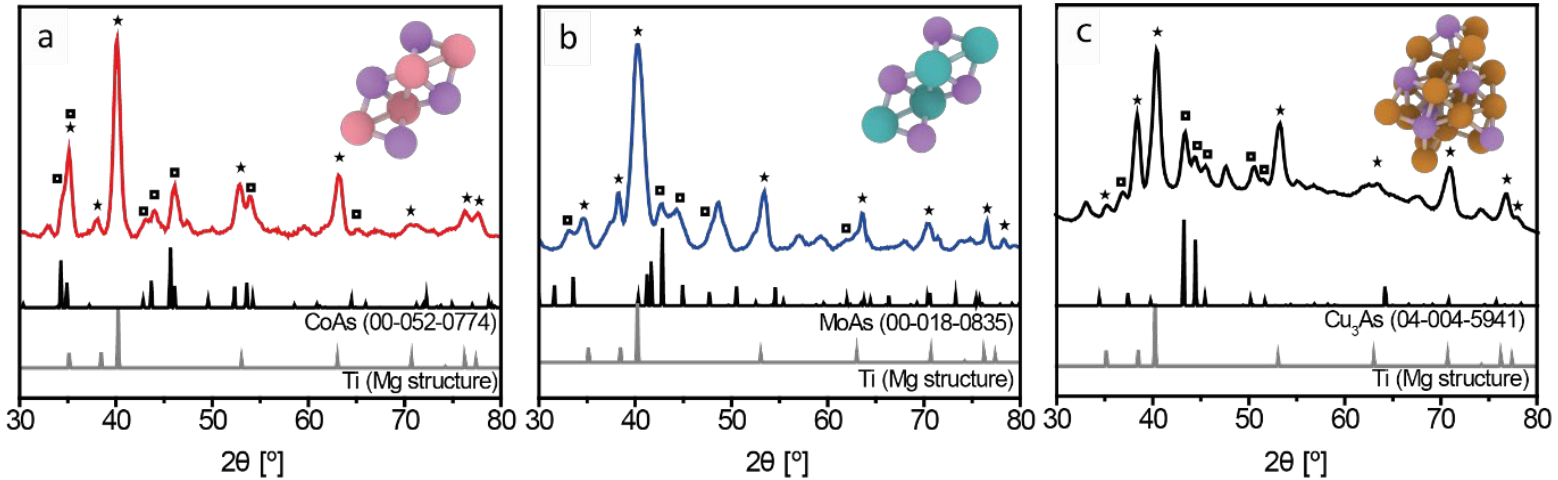

Figure 1: Physical characterisation of the transition metal arsenides. X-ray diffraction spectra of the synthesized (a) CoAs, (b) MoAs, and (c) $\mathrm{Cu}_{3}$ As thin films. Reference XRD spectra for the relevant arsenide and titanium support are also shown. The titanium substrate peaks (00-054-1294) are labeled with stars, the predominant TMAs phases are labelled with squares. The inserts show the crystal structures. 

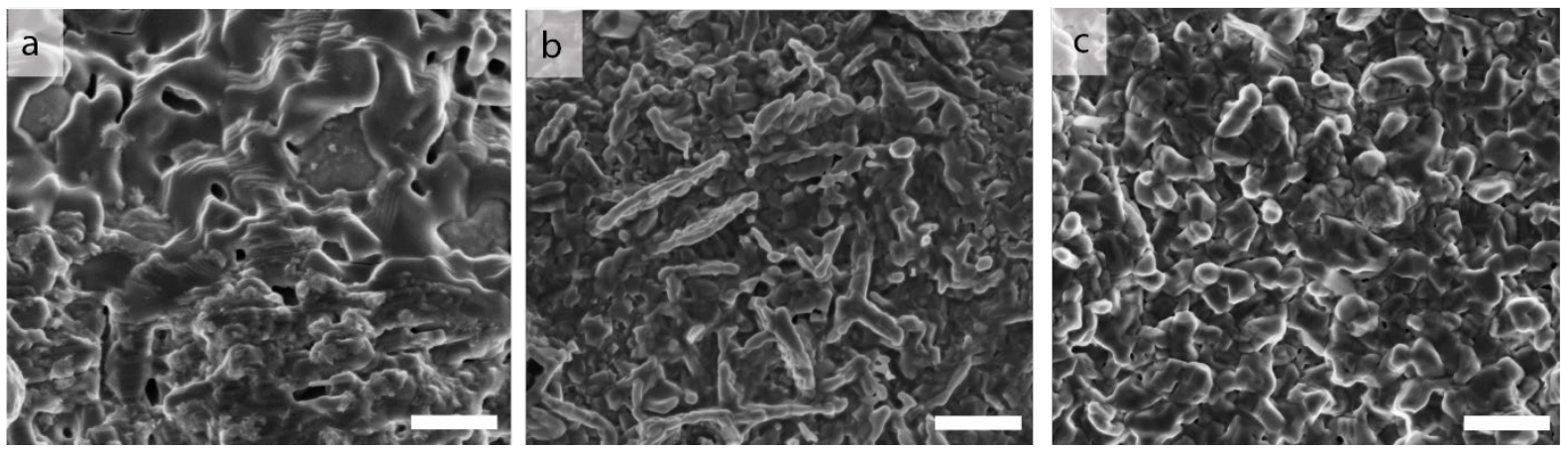

Figure 2: Scanning electron microscopy (SEM) micrographs of the synthesized (a) CoAs, (b) MoAs, and (c) $\mathrm{Cu}_{3}$ As thin films. The scale bar is $1 \mu \mathrm{m}$ for all of the images.

\section{Catalytic Activity and Stability}

A 3-electrode electrochemical cell was used to assess the catalytic activity of the synthesized TMAs films as well as the Ti-foil substrate. All three TMAs showed good activity for the HER (Figure 2a). MoAs demonstrated the best geometric current density with $303 \mathrm{mV}$ overpotential required to reach $-10 \mathrm{~mA} \cdot \mathrm{cm}^{-2}$. The overall order of geometric activity for the TMAs films was MoAs $>\mathrm{CoAs}>\mathrm{Cu}_{3} \mathrm{As}$. The Ti foil substrate demonstrated poor activity for the HER, reaching $-10 \mathrm{~mA} . \mathrm{cm}^{-2}$ at $-0.767 \mathrm{~V}$ vs. RHE. It is of note that due to the conductive nature of our $\mathrm{Ti}$ substrate, 4-point probe measurements on the as-synthesised catalysts could not be conducted to assess the conductivity of the TMAs catalysts. Although we did not measure the conductivity of the synthesized catalysts, Figure 2 a clearly demonstrates that the films are sufficiently conductive for this application, reaching current densities of up to $-15 \mathrm{~mA} \cdot \mathrm{cm}^{-2}$ at reasonable overpotentials. This matches what would be expected given literature electrical conductivity values for transition metal arsenides. ${ }^{37,38}$

To further investigate the activity of the catalysts, Tafel slopes were calculated for the synthesized TMAs catalysts (Table S2, Figure S3). The average Tafel slopes were 74, 76, and $123 \mathrm{mV} \cdot \mathrm{dec}^{-1}$ for MoAs, CoAs, and $\mathrm{Cu}_{3} \mathrm{As}$ respectively. These values are higher than that for the transition metal phosphides (typically $50-60 \mathrm{mV} \cdot \mathrm{dec}^{-1}$ ) and platinum $\left(30 \mathrm{mV} \cdot \mathrm{dec}^{-1}\right)$. The higher Tafel slopes indicate that the Volmer step may be the rate determining step rather than Tafel and Heyrovsky steps. However, while these values can be used as a guide for the identification of HER mechanisms, the Tafel analysis is based on a set of assumptions that do not universally hold. Indeed, factors such as conductivity, solvent environment and surface defects are known to complicate analysis of the Tafel kinetics. The electrochemical surface area (ECSA) for each of the synthesized TMAs films was also estimated (details provided in the supporting information). The HER activity normalized by the ECSA showed similar trends to the geometric activities (Figure S3) whereby MoAs and CoAs are shown to be more active than $\mathrm{Cu}_{3} \mathrm{As}$. 

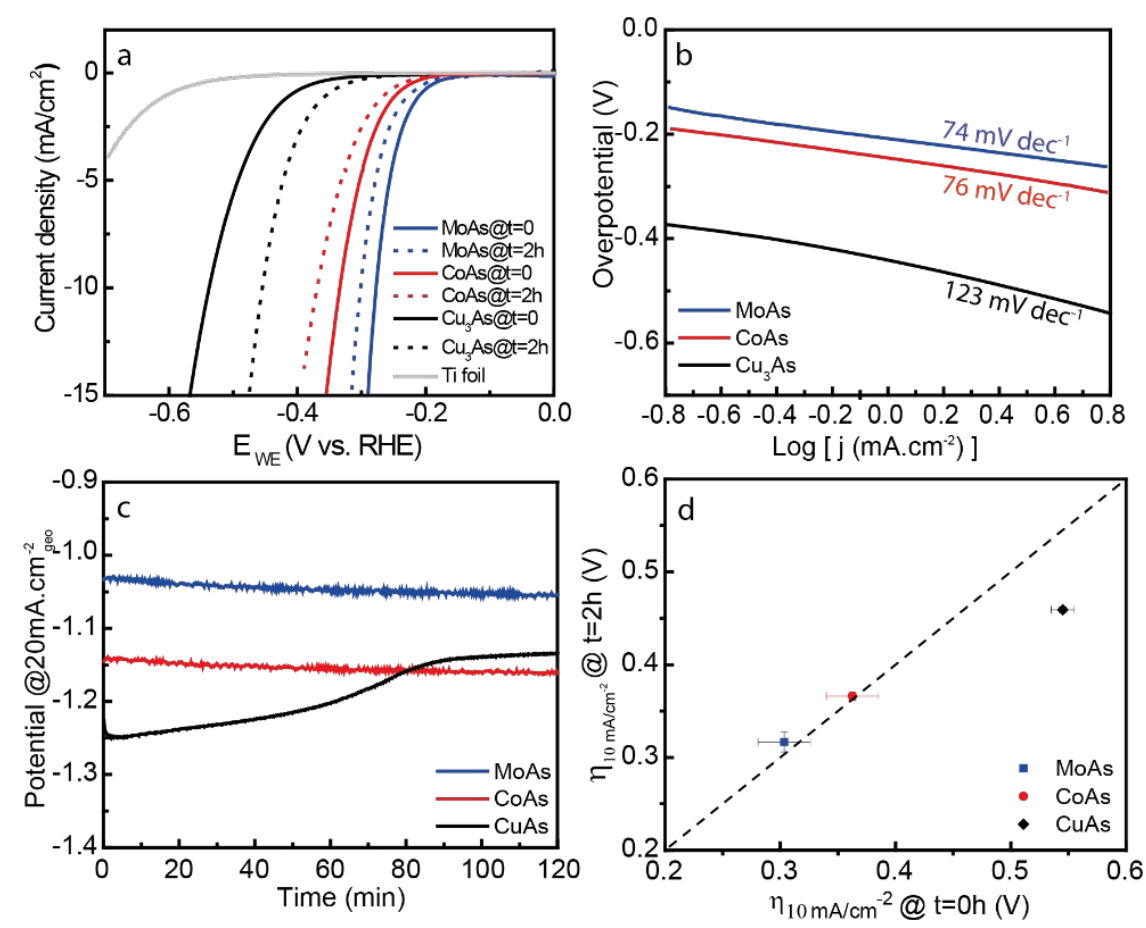

Figure 3: Electrochemical characterization of the TMAs catalysts. (a) Initial (solid lines), and postchronopotentiometry hold (dashed lines) iR-corrected linear sweep voltammograms with current normalized by geometrical surface area. (b) Tafel plots for the synthesized TMAs catalysts. (c) Chronopotentiometry measurements at $20 \mathrm{~mA} \mathrm{~cm}$ geo $^{-2}$ (d) The initial overpotential (@-10 mA.cm ${ }^{-2}$ geo $)$ plotted as a function of the post-

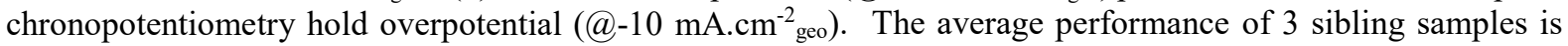
plotted with error bars representing the standard deviation. The diagonal dashed line is the response for a stable catalyst.

Towards understanding the experimental trends observed in the HER activity, we performed DFT calculations to determine the differential hydrogen adsorption free energies, $\Delta G_{\mathrm{H}}$. This method has previously shown that $\Delta G_{\mathrm{H}}$ is a good descriptor for HER activity on transition metals, ${ }^{26}$ transition bimetallic alloys ${ }^{6}{ }^{\text {transition metal sulfides }}{ }^{10,11}$ and transition metal phosphides. ${ }^{19}$ Specifically, $\Delta G_{\mathrm{H}}$ values that are closest to $0 \mathrm{eV}$ have previously shown to demonstrate the highest intrinsic activity for the HER. Details regarding the calculation of $\Delta G_{\mathrm{H}}$ can be found in the supplementary information. In brief, we use the theoretically determined limiting potential $U_{\mathrm{L}}$ as an estimate of activity, defined as the potential where all steps in the HER mechanism become exergonic. For the HER, a two coupled proton-electron transfer process, this is simply defined as $U_{\mathrm{L}}=-\frac{\left|\Delta G_{\mathrm{H}}\right|}{e}$, and so activity is maximized at $U_{\mathrm{L}}=$ $0, \Delta G_{\mathrm{H}}=0$. $U_{\mathrm{L}}$ has been shown to correlate well with experimental trends in activity for a number of processes. ${ }^{6,7,10,11,19,26}$. We utilized a Pourbaix analysis to determine the likely surface coverage and arrive at a $U_{\mathrm{L}}$ that accounts for $* \mathrm{O}$ or $* \mathrm{OH}$ poisoning of active sites, since some of the surfaces investigated interact strongly with water.

The BFDH algorithm ${ }^{34}$ was used to determine likely crystal facets, based on the geometry of the primitive unit cell. Calculated surface energies, seen in Figure S1, were all within $0.1 \mathrm{eV}$ and so every facet was tested for activity. Initial calculations screened the various crystal facets for each TMAs catalyst to determine the most active facet (CoAs (100), MoAs (100) and $\mathrm{Cu}_{3} \mathrm{As}$ (010)). Pourbaix diagrams were then calculated for these identified active facets (Figure 3). Lattice parameters and images of the surface termination for each material and facet are shown in Table S3. 
Figure 4a shows the calculated Pourbaix diagram for the CoAs (100) facet. At $0 \mathrm{~V}$ vs RHE, the dominant coverage on CoAs $(100)$ is a single ${ }^{*} \mathrm{OH}$ bound to the surface. However, as the potential is made increasing negative and reaches $-0.18 \mathrm{~V}$ vs. RHE, the bound $* \mathrm{OH}$ species is displaced by $* \mathrm{H}$, which then becomes active for HER through a Volmer-Heyrovsky mechanism. The binding energy of $\mathrm{H}^{*}$ on this surface is $\Delta G_{\mathrm{H}}=-0.18 \mathrm{eV}$. Overall, this gives a corresponding limiting potential $U_{\mathrm{L}}=-0.18 \mathrm{~V}$. As the potential is made even more negative, the dominant surface coverage shifts to having two bound ${ }^{*} \mathrm{H}$ in the unit cell.
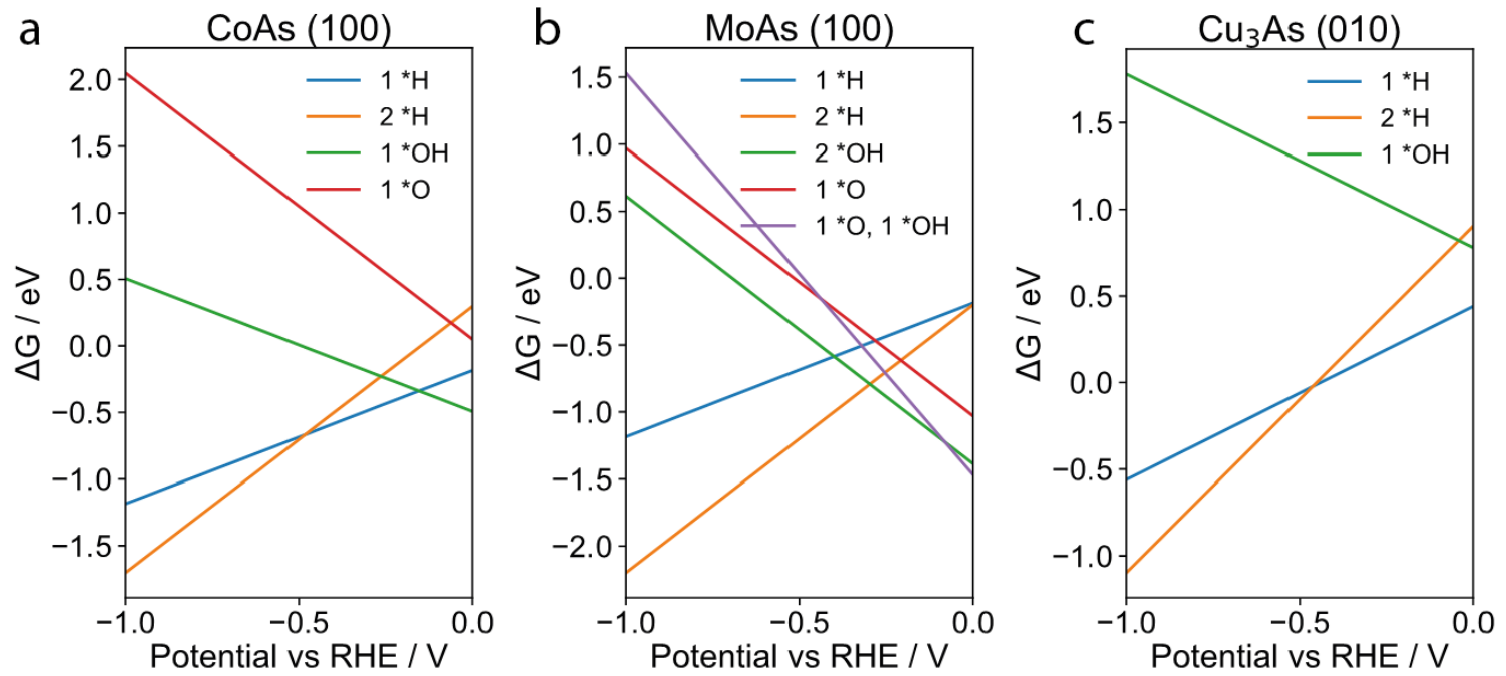

Figure 4: Pourbaix Diagram for most active TMA facets. (a) CoAs (100). (b) MoAs (100). (c) $\mathrm{Cu}_{3} \mathrm{As}$ (010). Each line in the legends denote the surface coverage. For example, $2 * \mathrm{H}$ corresponds to two bound hydrogens in the unit cell, while $1 * \mathrm{O}, 1 * \mathrm{OH}$ correponds to having both a bound oxygen and a bound hydroxyl in the unit cell.

Figure $4 \mathrm{~b}$ and $4 \mathrm{c}$ show the calculated Pourbaix diagram for the most active facets of MoAs and $\mathrm{Cu}_{3} \mathrm{As}$, respectively. A limiting potential of $U_{\mathrm{L}}=-0.09 \mathrm{~V}$ vs RHE for MoAs (100), and $U_{\mathrm{L}}=$ $-0.44 \mathrm{~V}$ vs $\mathrm{RHE}$ for $\mathrm{Cu}_{3} \mathrm{As}(010)$ was calculated.

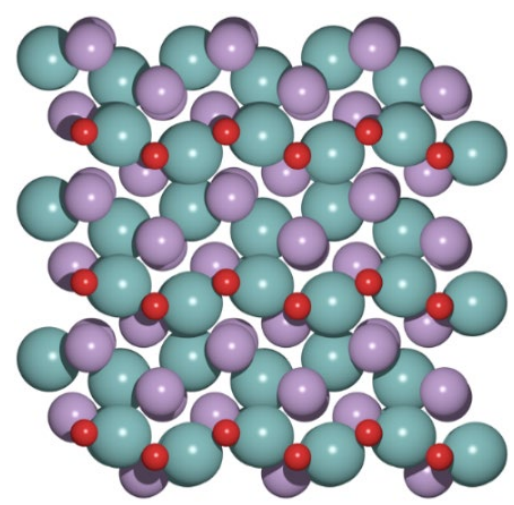

Figure 5: Stable surface termination of MoAs (100) surface. Purple spheres represent arsenic atoms, with bluegreen representing molybdenum, and red oxygen.

The stable coverage on MoAs (100) is an oxygen terminated surface illustrated in Figure 5. There, hydrogen adsorbs onto ${ }^{*} \mathrm{O}$ forming ${ }^{*} \mathrm{OH}$, from which HER occurs. $\mathrm{Cu}_{3} \mathrm{As}$ does not strongly bind oxygen containing compounds, where HER occurs at a relatively low coverage of hydrogen. The Pourbaix diagram for $\mathrm{Cu}_{3}$ As shows a simpler case, where the surface is fairly 
weak binding, meaning no competition with bound oxygen species occurs, and HER occurs at a low coverage.

Calculated Pourbaix diagrams and $U_{\mathrm{L}}$ for the remaining facets of the three materials can be found in the supporting information Figure S4 and Table S4. Figure 6 shows the comparison between experiment and theoretically determined descriptors. On the y-axis are (cf. Figure 3c) the experimentally measured overpotentials required to reach a current density of $-10 \mathrm{~mA} \mathrm{~cm}-$ ${ }^{2}$, and on the $\mathrm{x}$-axis are the theoretically determined binding free energies of hydrogen. Volcano plots were also prepared for current densities of 2.5 and $15 \mathrm{~mA} \mathrm{~cm}^{-2}$ revealing similar trends between the three materials (Figure S5). A volcano shape emerges from the three points (the solid black line is a line of best fit to guide the eye), suggesting the consistency between experimental and theoretical activities.

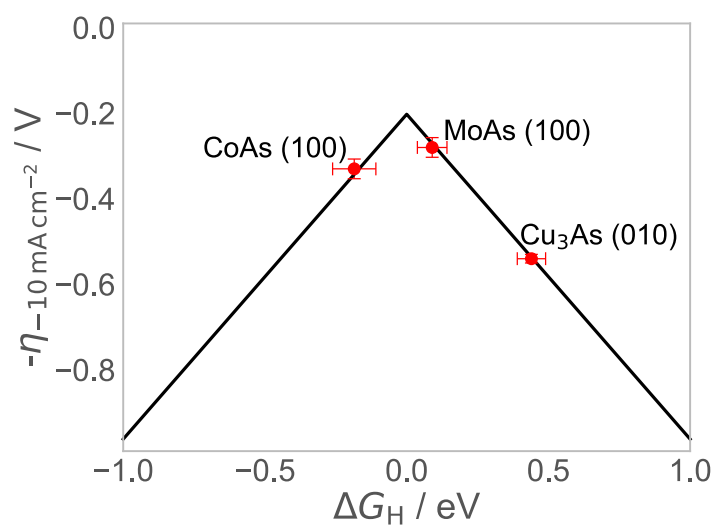

Figure 6: HER volcano plot for transition metal arsenides plotting the experimentally measured overpotential required to reach $-10 \mathrm{~mA} \mathrm{~cm}^{-2}$ as a function of the calculated binding energy of hydrogen on the catalyst surface $\left(\Delta G_{H}\right)$. Error bars on the $\mathrm{x}$-axis represent the standard deviation as estimated by the BEEF ensemble. Error bars on the y-axis represent the standard deviation among 3 repeated experiments. The solid line is shown to guide the eye.

To evaluate the stability of the TMAs catalysts, $2 \mathrm{~h}$ chronopotentiometry holds at current densities of $-20 \mathrm{~mA} \mathrm{~cm}^{-2}$ geo were performed (Figure 3c). The potential required to reach 10 $\mathrm{mA} \mathrm{cm}{ }^{-2}$ geo was assessed before and after the chronopotentiometry hold by LSVs (Figure $2 \mathrm{~d}$ ). Both the MoAs and CoAs are found to be relatively stable, with just a $4 \mathrm{mV}$ and $13 \mathrm{mV}$ loss in potential required to reach $10 \mathrm{~mA} \mathrm{~cm}^{-2}$ geo respectively. We speculate that one of the following factors could lead to an improvement such as this: (1) the reductive removal of a surface oxide resulting in a more conductive surface, (2) the roughening of the catalyst surface during electrochemical testing resulting in an increased surface area. A calculation was performed to confirm that the amount of current passed during the $2 \mathrm{~h}$ stability test is two orders of magnitude greater than that required to electrochemically corrode the films during the durability measurement (see Supporting Information for details). Conversely, the $\mathrm{Cu}_{3} \mathrm{As}$ samples on average gain $85 \mathrm{mV}$ in the potential required to reach $-10 \mathrm{~mA} \mathrm{~cm}{ }^{-2}$ geo. These differences in stability observed for the three TMAs catalysts is indeed an intriguing observation. SEM was performed post electrochemical testing (Figure S6) and revealed that surface rearrangements/morphological changes did occur during the stability measurements. In particular, $\mathrm{Cu}_{3} \mathrm{As}$, showed the appearance of smaller nanoparticle features $(50-100 \mathrm{~nm})$ post stability testing. This finding is supported by theory, where only $\mathrm{Cu}_{3} \mathrm{As}$ was found to have favorable vacancy formation energies (about $1 \mathrm{eV}$ downhill when moving two copper atoms 
from the (010) unit cell to bulk copper); the vacancies were included in the investigation of HER activity.

\section{Conclusions}

In conclusion, we investigated a previously unexplored class of materials for the HER, using a combined theoretical and experimental approach. Consistent with previous studies of similar ionic compounds, we find that trends in experimental activity agree with trends in the hydrogen binding energy, a simple descriptor for HER activity. We find that two of the synthesized transition metal arsenides, MoAs and CoAs, exhibit a moderate overpotential on a normalized electrochemical surface area basis. We further find that CoAs binds hydrogen too strongly, while MoAs is on the weak binding side of the volcano, which suggests an alloy may lie closer to the peak of the volcano and show higher HER activity. On MoAs, the poisoning of the surface by $* \mathrm{O}$ indicates that HER proceeds through the oxidized surface. $\mathrm{Cu}_{3} \mathrm{As}$ exhibits a large overpotential, due to a relatively weak binding energy of $\mathrm{H}$, and likely undergoes some surface reconstruction due to the instability of the surface relative to bulk copper.

Our analysis here was based on a purely thermodynamic approach, which implicitly assumes the associated activation barriers to scale similarly with binding energies regardless of binding site. This work was exploratory in nature and does not represent an exhaustive investigation of this class of materials. Future work will investigate trends in activation energies on additional arsenide candidates, and a broad spectrum of ionic materials

\section{Acknowledgments}

The authors gratefully acknowledge support from the Office of Basic Energy Sciences of the U.S. Department of Energy to the SUNCAT Center for Interface Science and Catalysis. LAK would like to thank the Toyota Research Institute. This work also presents results from a project sponsored by the Office of Energy Efficiency and Renewable Energy, Fuel Cell Technologies Office, Award number DE-EE0008084, competitively-selected under the solicitation "Topic 2A: Durable, High-Performance Materials and Interfaces for Advanced Water Splitting" (DE-FOA-0001647). Part of this work (SEM and XRD) was performed at the Stanford Nano Shared Facilities (SNSF), supported by the National Science Foundation under award ECCS-1542152. F.T.S acknowledges the National Science Foundation under award \#1265721. Some of the computing for this project was performed on the Sherlock cluster. We would like to thank Stanford University and the Stanford Research Computing Center for providing computational resources and support that contributed to these research results.

\section{Supporting Information Available}

The Supporting Information is available free of charge on the ACS Publications website.

Details of the Tafel slopes, electrochemical active surface area methods, and the DFT calculated formation energies, surface energetics, Pourbaix diagrams, theory determined limiting potentials, and SEM micrographs post electrochemical testing. Additionally, raw data plotted in Figures $1-6$ are tabulated. 


\section{References}

(1) U.S. Energy Information Administration, . The Impact of Increased Use of Hydrogen on Petroleum Consumption and Carbon Dioxide Emissions; 2008.

(2) Barbir, F. PEM Electrolysis for Production of Hydrogen from Renewable Energy Sources. Sol. Energy 2005, 78, 661-669.

(3) Seh, Z. W.; Kibsgaard, J.; Dickens, C. F.; Chorkendorff, I.; Nørskov, J. K.; Jaramillo, T. F. Combining Theory and Experiment in Electrocatalysis: Insights into Materials Design. Science (80-. ). 2017, 355, eaad4998.

(4) Ginley, D. S.; Cahen, D. Fundamentals of Materials for Energy and Environmental Sustainability; Cambridge University Press, 2011.

(5) Press, R. J.; Santhanam, K. S. V.; Miri, M. J.; Bailey, A. V.; Takacs, G. A. Introduction to Hydrogen Technology; Wiley-Interscience: Hoboken, 2008.

(6) Greeley, J.; Nørskov, J. K.; Kibler, L. A.; El-aziz, A. M.; Kolb, D. M. Hydrogen Evolution Over Bimetallic Systems: Understanding the Trends. ChemPhysChem 2006, 7, 1032-1035.

(7) Greeley, J.; Jaramillo, T. F.; Bonde, J.; Chorkendorff, I.; Nørskov, J. K. Computational High-Throughput Screening of Electrocatalyticmaterials for Hydrogen Evolution. Nat. Mater. 2006.

(8) Marković, N. M.; Grgur, B. N.; Ross, P. N. Temperature-Dependent Hydrogen Electrochemistry on Platinum Low-Index Single Crystal Surfaces in Acid Solutions. $J$. Phys. Chem. B 1997, 101, 5405-5413.

(9) Vesborg, P. C. K.; Jaramillo, T. F. Addressing the Terawatt Challenge: Scalability in the Supply of Chemical Elements for Renewable Energy. RSC Adv. 2012, 2, 7933.

(10) Hinnemann, B.; Moses, P. G.; Bonde, J.; Jørgensen, K. P.; Nielsen, J. H.; Horch, S.; Chorkendorff, I.; Nørskov, J. K. Biomimetic Hydrogen Evolution : MoS2 Nanoparticles as Catalyst for Hydrogen Evolution. J. Am. Chem. Soc. 2005, 127, 5308-5309.

(11) Jaramillo, T. F.; Jørgensen, K. P.; Bonde, J.; Nielsen, J. H.; Horch, S.; Chorkendorff, I. Identification of Active Edge Sites for Electrochemical H 2 Evolution From. Science (80-. ). 2007, 317, 100-103.

(12) Wang, H.; Yuan, H.; Sae Hong, S.; Li, Y.; Cui, Y. Physical and Chemical Tuning of Two-Dimensional Transition Metal Dichalcogenides. Chem. Soc. Rev. 2015, 44, 2664 2680.

(13) Pumera, M.; Sofer, Z.; Ambrosi, A. Layered Transition Metal Dichalcogenides for Electrochemical Energy Generation and Storage. J. Mater. Chem. A 2014, 2, 89818987.

(14) Saadi, F. H.; Carim, A. I.; Velazquez, J. M.; Baricuatro, J. H.; McCrory, C. C. L.; Soriaga, M. P.; Lewis, N. S. Operando Synthesis of Macroporous Molybdenum Diselenide Films for Electrocatalysis of the Hydrogen-Evolution Reaction. ACS Catal. 2014, 4, 2866-2873.

(15) Kong, D.; Wang, H.; Lu, Z.; Cui, Y. CoSe2 Nanoparticles Grown on Carbon Fiber Paper: An Efficient and Stable Electrocatalyst for Hydrogen Evolution Reaction. $J$. Am. Chem. Soc. 2014, 136, 4897-4900.

(16) Seh, Z. W.; Fredrickson, K. D.; Anasori, B.; Kibsgaard, J.; Strickler, A. L.; 
Lukatskaya, M. R.; Gogotsi, Y.; Jaramillo, T. F.; Vojvodic, A. Two-Dimensional Molybdenum Carbide (MXene) as an Efficient Electrocatalyst for Hydrogen Evolution. ACS Energy Lett. 2016, 1, 589-594.

(17) Vrubel, H.; Hu, X. Molybdenum Boride and Carbide Catalyze Hydrogen Evolution in Both Acidic and Basic Solutions. Angew. Chemie - Int. Ed. 2012, 51, 12703-12706.

(18) Chen, W.-F.; Muckerman, J. T.; Fujita, E. Recent Developments in Transition Metal Carbides and Nitrides as Hydrogen Evolution Electrocatalysts. Chem. Commun. 2013, $49,8896$.

(19) Kibsgaard, J.; Tsai, C.; Chan, K.; Benck, J. D.; Nørskov, J. K.; Abild-Pedersen, F.; Jaramillo, T. F. Designing an Improved Transition Metal Phosphide Catalyst for Hydrogen Evolution Using Experimental and Theoretical Trends. Energy Environ. Sci. 2015, 8, 3022-3029.

(20) Xiao, P.; Sk, M. A.; Thia, L.; Ge, X.; Lim, R. J.; Wang, J.-Y.; Lim, K. H.; Wang, X. Molybdenum Phosphide as an Efficient Electrocatalyst for the Hydrogen Evolution Reaction. Energy Environ. Sci. 2014, 7, 2624-2629.

(21) Kibsgaard, J.; Jaramillo, T. F. Molybdenum Phosphosulfide: An Active, Acid-Stable, Earth- Abundant Catalyst for the Hydrogen Evolution Reaction. Angew. Chemie - Int. Ed. 2014, 53, 14433-14437.

(22) McEnaney, J. M.; Chance Crompton, J.; Callejas, J. F.; Popczun, E. J.; Biacchi, A. J.; Lewis, N. S.; Schaak, R. E. Amorphous Molybdenum Phosphide Nanoparticles for Electrocatalytic Hydrogen Evolution. Chem. Mater. 2014, 26, 4826-4831.

(23) Benck, J. D.; Hellstern, T. R.; Kibsgaard, J.; Chakthranont, P.; Jaramillo, T. F. Catalyzing the Hydrogen Evolution Reaction ( HER) with Molybdenum Sulfide Nanomaterials. ACS Catal. 2014, 4, 3957-3971.

(24) Parsons, R. The Rate of Electrolytic Hydrogen Evolution and the Heat of Adsorption of Hydrogen. Trans. Faraday Soc. 1957, 1053-1063.

(25) Conway, B. E.; Bockris, J. O. Electrolytic Hydrogen Evolution Kinetics and Its Relation to the Electronic and Adsorptive Properties of the Metal. J. Chem. Phys. $1957,26$.

(26) Nørskov, J. K.; Bligaard, T.; Ashildur, L.; Kitchin, J. R.; Chen, J. G.; Pandelov, S.; Stimming, U. Trends in the Exchange Current for Hydrogen Evolution. Electrochem. Soc. 2005, 152, 23-26.

(27) Nørskov, J. K.; Rossmeisl, J.; Logadottir, A.; Lindqvist, L.; Kitchin, J. R.; Bligaard, T.; Jónsson, H. Origin of the Overpotential for Oxygen Reduction at a Fuel-Cell Cathode. J. Phys. Chem. B 2004, 108, 17886-17892.

(28) Wellendorff, J.; Lundgaard, K. T.; Møgelhøj, A.; Petzold, V.; Landis, D. D.; Nørskov, J. K.; Bligaard, T.; Jacobsen, K. W. Density Functionals for Surface Science:

Exchange-Correlation Model Development with Bayesian Error Estimation. Phys. Rev. $B$ - Condens. Matter Mater. Phys. 2012, 85, 32-34.

(29) Giannozzi, P.; Baroni, S.; Bonini, N.; Calandra, M.; Car, R.; Cavazzoni, C.; Ceresoli, D.; Chiarotti, G. L.; Cococcioni, M.; Dabo, I.; et al. QUANTUM ESPRESSO: A Modular and Open-Source Software Project for Quantum Simulations of Materials. $J$. Phys. Condens. Matter 2009, 21, 395502.

(30) Bahn, S. R.; Jacobsen, K. W. An Object-Oriented Scripting Interface to a Legacy Electronic Structure Code. Comput. Sci. Eng. 2002, 4, 56-66. 
(31) Laasonen, K.; Car, R.; Lee, C.; Vanderbilt, D. Implementation of Ultrasoft Pseudopotentials in Ab Initio Molecular Dynamics. Phys. Rev. B 1991, 43, 6796-6799.

(32) Vanderbilt, D. Soft Self-Consistent Pseudopotentials in a Generalized Eigenvalue Formalism. Phys. Rev. B 1990, 41, 7892-7895.

(33) Laasonen, K.; Pasquarello, A.; Car, R.; Lee, C.; Vanderbilt, D. Car-Parrinello Molecular Dynamics with Vanderbilt Ultrasoft Pseudopotentials. Phys. Rev. B 1993, 47, 10142-10153.

(34) Docherty, R.; Clydesdale, G.; Roberts, K. J.; Bennema, P. Application of BravaisFriedel-Donnay-Harker, Attachment Energy and Ising Models to Predicting and Understanding the Morphology of Molecular Crystals. J. Phys. D. Appl. Phys. 2000, 24, 89-99.

(35) Gunn, B. Y. S. R. The Enthalpies of Formation of Arsine and Biarsinel. 1972, 11, 796799.

(36) Miedema, A, R. On the Heat of Formation of Solid Alloys. J. Less Common Met. 1975, 41, 283-298.

(37) Senzaki, Y.; Gladfelter, W. L. Molecular Routes to Cobalt Arsenide and Cobalt Phosphide Thin Films. Comparison of a Two-Source and a Single-Source Chemical Vapour Deposition. Polyhedron 1994, 13, 1159-1167.

(38) Pauwels, L. J.; Maervoet, G.; Vervaeke, R. Electrical Resistivity and Magnetic Susceptibility Measurements on Copper Arsenides. Z. anorg. allg. Chem. 1973, 397, 307-313. 


\section{TOC Image}

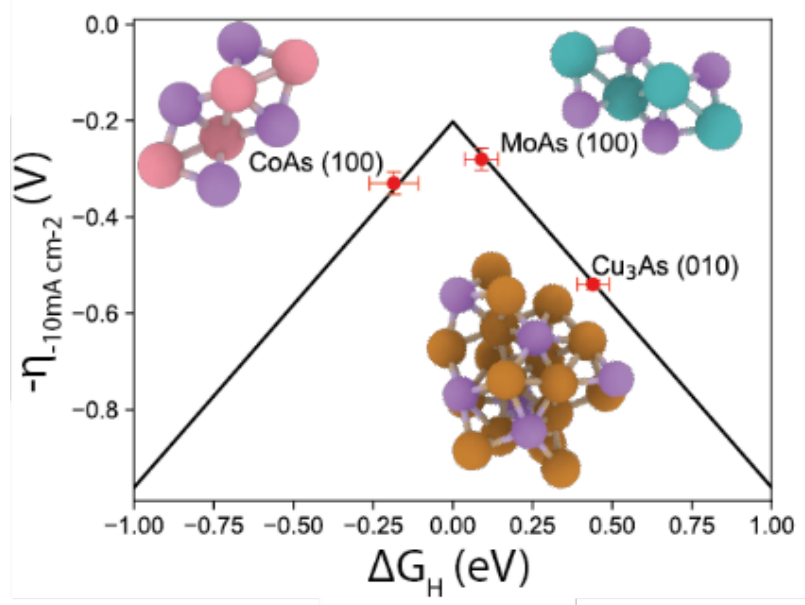

